\title{
A Systematic Review on Complex Networks and its Performance Evaluation Metrics
}

\author{
Selasie A. Brown \\ University of Professional \\ Studies, Accra \\ P.O. Box LG 149 \\ Accra-Ghana
}

\author{
Patrick Kudjo \\ University of Professional \\ Studies, Accra \\ P.O. Box LG 149 \\ Accra-Ghana
}

\author{
Francis Amavi \\ Datalink Institute \\ Community 10 New Road \\ P.O. Box CO 2481 \\ Tema
}

\begin{abstract}
The rapid advancement in computer systems has generated an increasing interest in the study and analysis of complex networks and its applications in the real world. Previous studies on complex networks have mainly been focused on investigating and presenting theories and concepts that explain the basic models such as regular graphs, random graphs, small-world networks, and scale-free networks with its application in the field of business, sociology, biology, immunology, and technology. In order to better comprehend the recent progress in complex systems, this paper reviews extensively, the current progress made towards complex network analysis by exploring four core aspects of complex network. The fundamental aspects reviewed are Random Graph, Scale-Free, Biological Networks, and Small World. Additionally, an in-depth analysis of the theoretical models, tools, techniques, and evaluation metrics applied in previous studies are presented. Finally, the paper provides a thorough analysis of complex network, report some related works, and attempt to address some challenges associated with using discrete-event simulators for performance evaluation of complex networks.
\end{abstract}

\section{General Terms}

Complex Network, Small Networks Random Graph

\section{Keywords}

Complex Network, Random Graph, Biological Networks, Small World Social Network, Network Simulators

\section{INTRODUCTION}

The study of complex systems is an active area of scientific research inspired largely by the empirical study of real-world networks such as social networks, biological networks, technological networks, semantic networks, computer networks, World Wide Web, and Food webs [1].

It is a widely accepted set of theory that evaluates networks evolved mainly in the domain of a branch of discrete mathematics known as graph theory. To the best of our knowledge, the concept was first investigated by the Swiss mathematician Leonhard Euler [2-5]. A network is defined as a collection of entities that are interconnected with links, where the entities represent vertices and the edges of the links. Newman [6] defined a network as a set of items called vertices or nodes with connections between them known as edges, Newman reiterated in his study that systems taking the form of networks are called graphs. Similarly, Chen et al. [7] describe the process of representing large graphs in realworld situations as complex networks and further defined complex network as the non- trivial topological features that do not only occur in simple networks but often in graphs modelling of real systems.
In another related study, Boccaletti et al. [5] described the concept as systems whose network structure is irregular, complex, and dynamically evolving in time. More importantly, the concept has been applied in several domains of study such as brain connectivity, time series modelling, global cargo ship management, air transport management, etc. Based on these definitions and its application to several domains of studies, one can say that the study of complex network has significantly improved over the past decade as a result of the recent technological advancement in computer systems and the deeper understanding gained from previous studies on complex network analysis and investigation such as regular graphs, random graphs, small-world networks, and scale-free networks. Small world network according to Watts and Strogatz [8] is a class of networks that are "highly clustered, like regular lattices, yet have small characteristic path lengths, like random graphs" in order words most of the nodes have one or two links but a few nodes have a large number of links. Scale-free network, on the other hand, is one of the significant and most fascinating discoveries in complex network according to scholars in the complex network engineering domain [9]. Barabási and Albert [10] describe the scale-free network as networks whose connectivity distributions are in a power-law form that is independent of the network scale, in other words, the probability that a node is selected uniformly at random has a certain number of links (degree) follows a particular mathematical function called a power law. These models and other complex network models are presented in section two. A significant benefit that can be delivered from the evolution of complex networks [11] is the knowledge gained on how to represent biological networks, social networks, and technological networks using nodes and links. Thus, this paper surveys the current progress made towards complex network analysis by investigating four core aspects of complex network. Additionally, this study presents an indepth analysis of the theoretical models, the tools and techniques applied in previous studies [12-16].

\section{Organization of the Paper:}

The remaining sections of the paper are organized as follows. Section 2 presents a detailed analysis of the various theoretical models of complex networks. Section 3 presents an overview of the tools and techniques applied in previous studies for complex network analysis. The trends and development with regards to complex network analysis is presented in section 4 . Section 5 discusses some real-world applications of a complex network. Section 6 Discuses the use of discrete event simulators for network evaluation performance and makes recommendations for optimization Section 7 summaries and concludes the study. 


\section{COMPLEX NETWORK MODELS}

This section presents a brief overview of complex network models

\subsection{Small World Networks}

The concept of small-world network was first considered by a Hungarian writer Frigyes Karinthy in 1929. Stanley Milgram in 1967 experimental verified the small world phenomenon quantitatively by randomly selecting people from locations like Kansas or Nebraska, and tasked them to start a chain of letter-forwarding. The whole idea was to prove that, two arbitrary people are connected by only six degrees of separation, i.e. the diameter of the corresponding graph of social connections is not much larger than six. [17, 18]. In 1998, Duncan Watts and Steven Strogatz [8] published the first small-world network model, which through a single parameter smoothly interpolates between a random graph and a lattice. Their model demonstrated that with the addition of only a small number of long-range links, a regular graph, in which the diameter is proportional to the size of the network, can be transformed into a "small world" in which the average number of edges between any two vertices is very small (mathematically, it should grow as the logarithm of the size of the network), while the clustering coefficient stays large. According to their definition, a small world is a network where the typical distance L between two randomly chosen nodes grows proportionally to the logarithm of the number of nodes $\mathrm{N}$ in the network. [19,20].
$\mathrm{L} \propto \log \mathrm{N}$

It is known that a wide variety of abstract graphs exhibit the small-world property, e.g., random graphs and scale-free networks [8]. The two main properties used to identify smallworld networks are clustering among nodes, which is represented mathematically as fellows. [21, 22]

$$
\mathrm{C}_{i}=\frac{2_{\mathrm{ei}}}{\mathrm{K}_{\mathrm{i}}\left(\mathrm{K}_{\mathrm{i}} 1\right)}
$$

The clustering coefficient is a metric that represents the density of triangles in the network. For instance, sparse random graphs have a vanishingly small clustering coefficient, while real-world networks often have a coefficient significantly larger. Small-world networks also have short path lengths (L) in random networks; the path length is a measure of the distance between nodes in the network, calculated as the mean of the shortest geodesic distances between all possible node pairs [23]. Small world properties are found in many real-world phenomena, including websites with navigation menus, food chains, electric power grids, metabolite processing networks, networks of brain neurons, voter networks, telephone call graphs, and social influence networks. The diagram (fig 1 and fig 2) clearly depicts Watts- Strogatz Small World model large, clustering coefficient, and high average path length respectively.
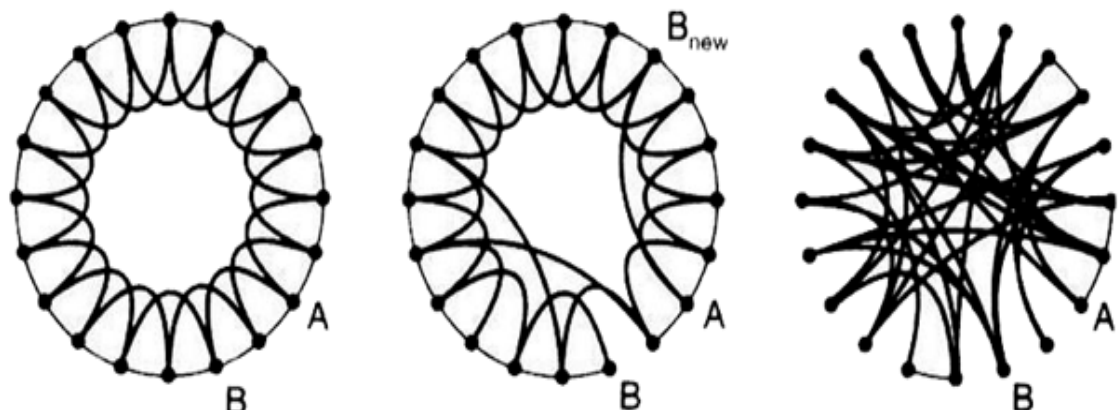

Fig: 1 Watt- Strogatz small world Network

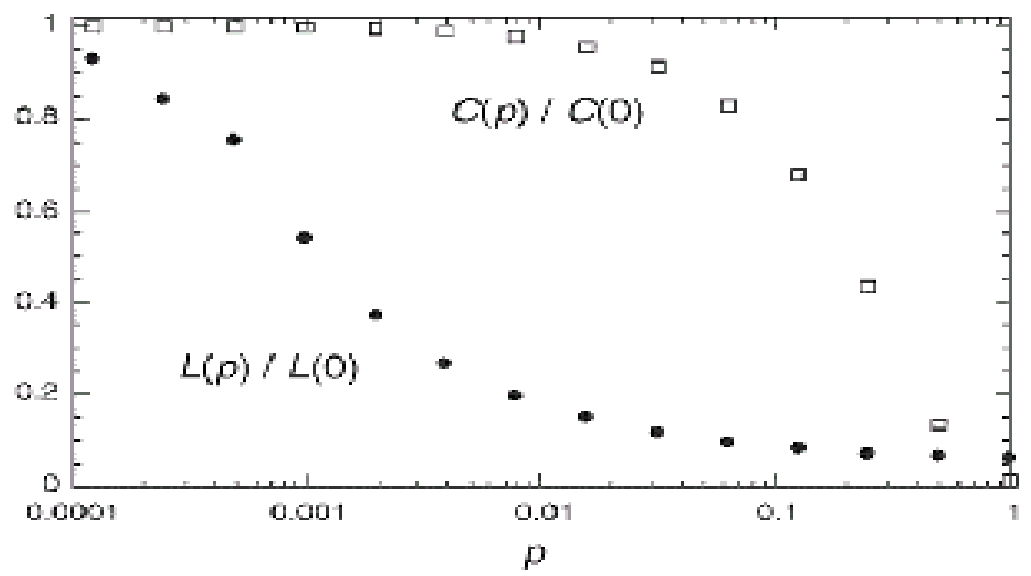

Fig: 2 Clustering Coefficient

\subsection{Small World Networks}

The scale-free network uses the Barabási-Albert (BA) Model [24]. It was empirically investigated in the late 1990s with the reporting of discoveries of power-law degree distributions in real-world networks. The BA model is mainly described as the preferential attachment; a concept that promotes the understanding of the properties of scalefree networks [10]. Tao et al. [25] describe the properties of the BA model as growth and preferential attachment. Growth was explained as a network that starts with a small number of $m_{o}$ of nodes, at every time step, a new node with 
$m\left(<=n_{0}\right)$ edges is added that connects the new node to $m$ different nodes already present in the system. The preferential attachment, on the other hand, was described as a situation it was assumed that the probability $\prod\left(\mathbf{K}_{\mathbf{i}}\right)$ that is, a new node was connected to node $i$ depended on the degree $k_{i}$ of node $i$ in such a way that

(3) are rest nodes of networks

$$
\Pi\left(\mathbf{K}_{\mathrm{i}}\right)=\frac{\mathbf{K}_{\mathrm{i}}}{\sum_{j} k_{j}, j}
$$

In another related study Zhang et al. [26] viewed the concept as a preferential attachment that produced several good deals of algorithms and analysis. The degree of the scale-free network follows power-law distribution [27] with a flat tail for large $\mathrm{k}$ or the power distribution law deviating at the tail. The formula and figure 3-4 respectively depict the model.

$$
\begin{aligned}
& p(k) \sim k^{-\gamma} \\
& p(k) \sim k^{-\gamma} e^{-\frac{k}{\kappa}}
\end{aligned}
$$

Most of the reported "power laws" fail when challenged with rigorous statistical testing, but the more general idea of heavy-tailed degree distributions which many of these networks do genuinely exhibit (before finite-size effects occur) are very different from what one would expect if edges existed independently and at random [10]. It usually increases with a small number of starting nodes $\left(n_{0}\right)$, at every time step, a new node $m\left(<=n_{0}\right)$ links was added that connect the new node to $\mathrm{m}$ different nodes already present in the system. When choosing the nodes to which the new node will be connected to node $\mathrm{i}$ depends on its degree ki. Networks with power-law degree distribution are highly resistant to the random deletion of vertices. They are also quite sensitive to targeted attacks aimed at fracturing the network quickly. While random graphs (ER) have an average distance of order $\log \mathrm{N}$ between nodes, where $\mathrm{N}$ is the number of nodes, a scale-free network on the other has a distance of $\log \mathrm{N}$ [28]. The nodes are highly connected than others in any real network. Some nodes are more highly connected than others in any real networks and they are used to quantify their effect. Theoretically, this model it can be expressed as a $p k$ where, $k$ is called the degree, and $p k$ is the degree distribution. The simplest random graph models [29] predict a bell-shaped Poisson distribution for $p k$ which is highly skewed and decays much more slowly than a Poisson for many real-world networks. The presence of two-point connectivity correlations does not alter the extreme weakness of scale-free network to epidemic diffusion and this result is related to the divergence of the nearest neighbor's average connectivity, a divergence that is ensured by the connectivity detailed balance condition, to be satisfied in physical networks [30]. The scale-free network has significantly impacted several types of research works in diverse fields of studies and has successfully been applied to stock market analysis, social networks, citation networks, study of sexually transmitted diseases. A distinctive characteristic of a scale-free network is the realization that the structure and the evolution of networks are inseparable [10]. William Graham in 2013 reported on the following features that help distinguish between a scale network and random networks.
- A scale-free network promotes high-speed transfer of information energy

- A scale-free network is robust because it can still function with a random removal of a few nodes

- The power law of scale-free network has a characteristic i.e. constant exponent which is known as a dimension. This implies that irrespective of the size of the network, the dimension remains the same.

- Any two arbitrary chosen nodes in a scale-free network can be connected via a few other intermediary nodes

- Scale-free network as promotes high-speed transfer of information or energy.

- A scale-free network with a limited number of nodes (hubs) improves heavily on connectivity.

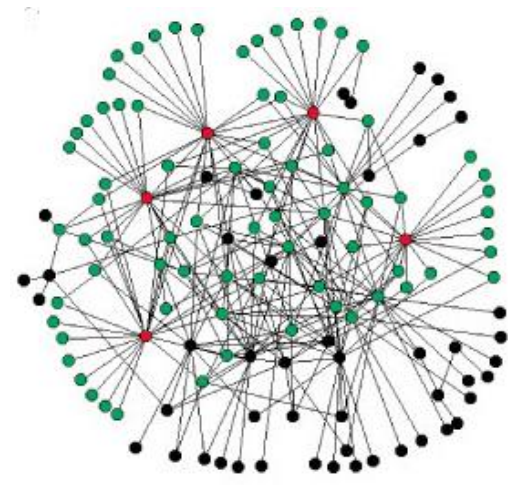

Fig: 5 A Scale-Free network

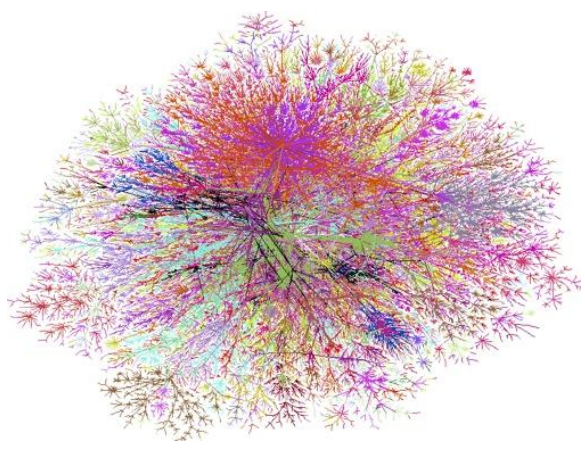

Fig: 6 Internet Routers of Scale-free network

\subsubsection{Random Graphs}

Erd"os and R'enyi [31] describe the random graph concept as a probabilistic construction of a graph with large girth and large chromatic number. A random graph model thus consists of $n$ nodes or vertices $(\mathrm{V})$, joined by links or edges (E) which are placed between pairs of vertices [32]. The set $\mathrm{V}$ and $\mathrm{E}$, therefore, define a graph $\mathrm{G}=(\mathrm{V}, \mathrm{E})$. The vertices of the random graph represent the objects to be model whiles the edges represent the relation between pairs of these objects E.N. Gilbert as well introduced the G (N, P) model of random graphs $[33,34]$ which shows that a statistical ensemble of a network, $G$, with two fixed parameters, is a given number of nodes $\mathrm{N}$ in each ensemble member and a given probability $\mathrm{p}$ that two nodes have an interconnecting link [34-36]. A graph can be represented as directed, undirected and weighted or non-weighted, a set of vertices $\mathrm{V}$ connected by edges $\mathrm{E}$ with no defined directionality or ordering is known as undirected graph whiles a set of vertices $\mathrm{V}$ connected by edges $\mathrm{E}$ with defined directionality is called a directed graph. Other related terms 
and concepts that have been investigated are Poisson random graphs, power-law degree distribution, bipartite graphs, degree correlations, exponential random graphs, and Markov graphs have been investigated [37-41].

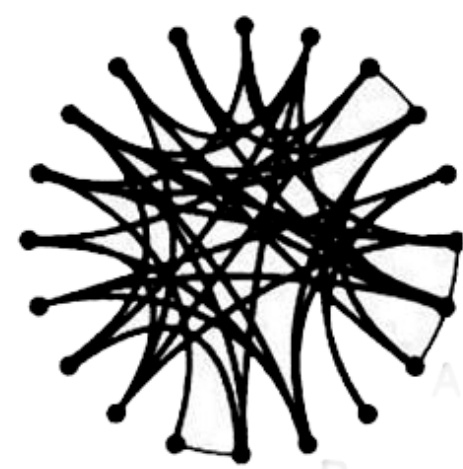

Fig. 7 Ra

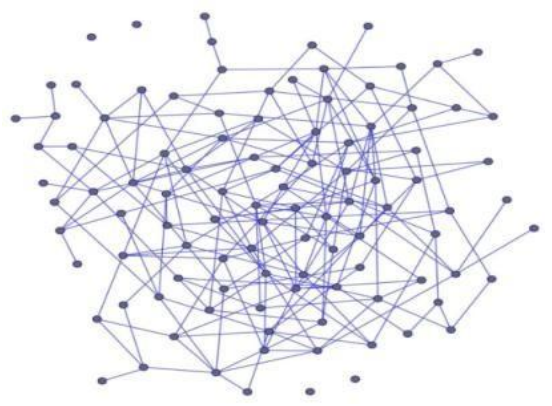

Fig. 8 Random Graph

\section{THEORETICAL TOOLS}

The major theoretical tools used in complex networks include graph model, statistical analysis, and probabilities analysis. More specifically, Centrifuge, Symmetrix, Cuttlefish, Cytoscape, EgoNet, Gephi, Graph-tool, GraphChi, Graphviz [42-44] are some of the tools that have been successfully applied in previous studies. For the purpose of this review, more emphasis will be put on Cytoscape and Gephi software.

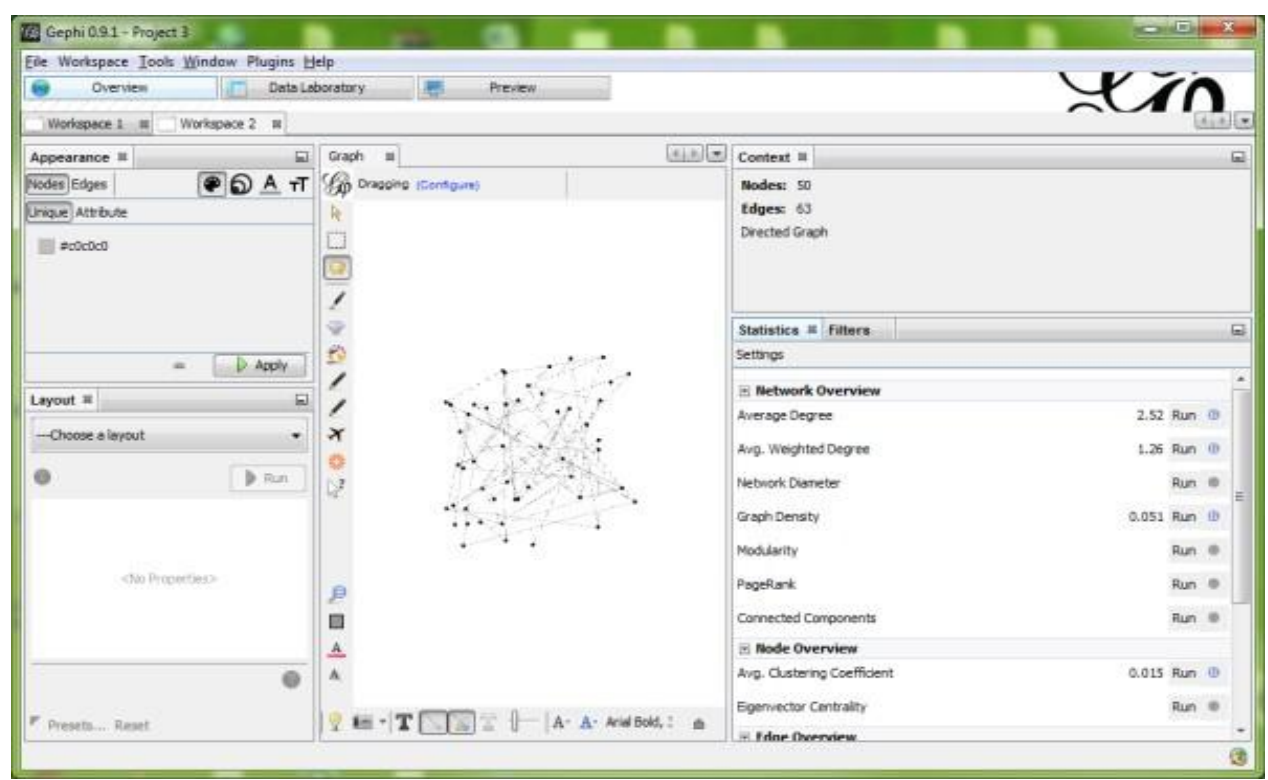

Fig: 9 Graphical User Interface (GUI) of Gephi software

Cytoscape is an open-source platform developed initially for biological research; nevertheless, it is currently applied in complex networks as a visualization tool. Shannon, Paul et al [45] used it to integrate models of biomolecular interaction networks. Gephi [46] is also an open-source for graphs network analysis. It uses a 3D render engine to display large networks in real-time and to speed up the exploration. Simulations are normally conducted using synthetic networks and real-world data. 


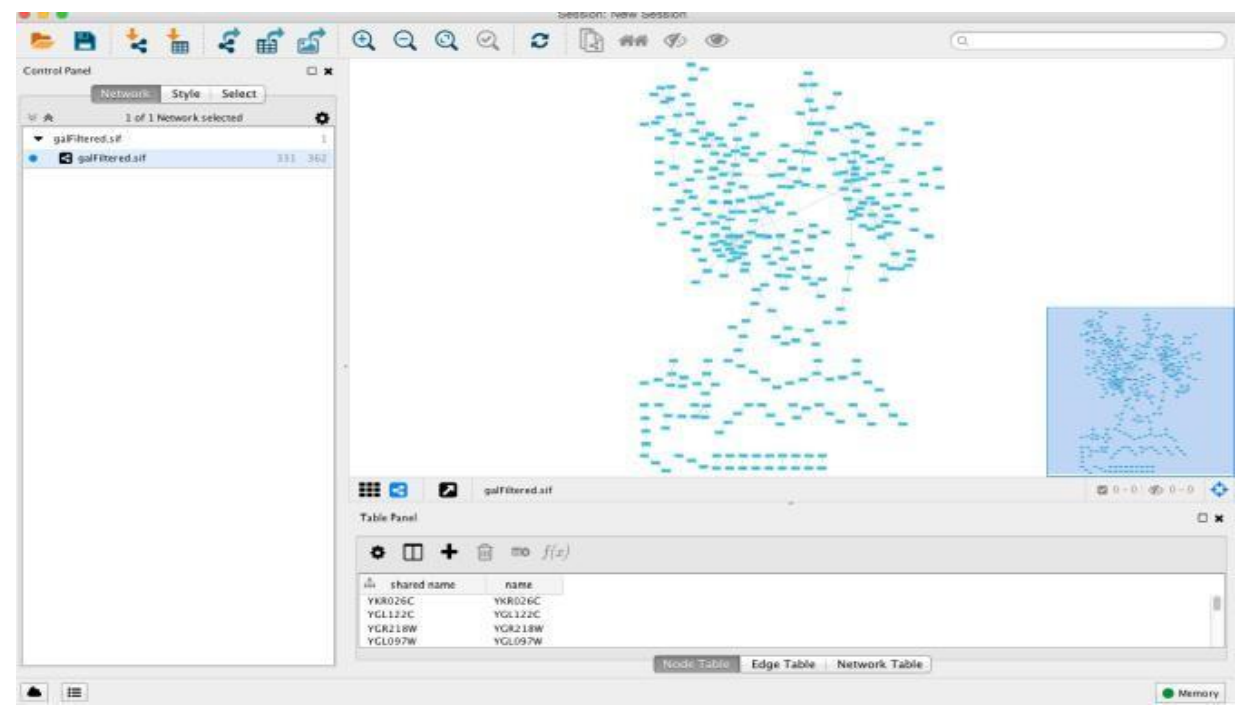

Fig 10 GUI of Cytoscape

node $i$ and node $j$, otherwise, aij=zero. In effect, all elements of aij of the adjacency matrix are either one or zero and A as a symmetric for undirected graphs, but not symmetric for directed graphs where there could be a link from $\mathrm{i} \rightarrow \mathrm{j}$, but not in the opposite direction $\mathrm{j} \rightarrow \mathrm{i}$. Undirected graphs are substantially easier to analyze because a symmetry of A leads to agreeable and powerful properties such as real eigenvalues which are generally complex with respect to non-symmetric matrix and eigenvectors [51, 52]. Li et al. [53] investigated the linear correlation coefficients between the mostly widely experimented metrics in complex networks with application in functional brain networks. The findings indicate that the metrics in each of the classes of graphs study above shows correlations and independences between metrics, this implies that it is possible to determine a small set of metrics by including only one metric from each subset of mutually strong dependent metrics. Again, a graph with a degree distribution as the unweighted functional brain network with a fixed threshold tends to possess a similar metric correlation pattern which in turn influences the degree distribution on metric relations.

\subsection{Social Network}

The empirical study on social network date back in the seventeenth century [54], Social network is a platform that provides a vast amount of information infrastructure for people to exchange ideas and discuss diverse topics. Before the introduction of the currently popular online social network such as Facebook, WeChat, Twitter, LinkedIn, WhatsApp, Reddit, Pinterest, etc., the social network was developed and adopted by the complex network community using real-world data [5, 11], which includes personal relations, music, collaborations, religion [55-59]. In this review, the researchers will only investigate online social networks as well as current research trends and challenges related to online social networks. A recent study on social networks focuses on social graph analysis; social network graphs represent a mathematical abstraction for modelling relationships between things (nodes) and edges (relationships) [60]. Empirical studies conducted on social graph analysis reveals a domain-specific analysis, this means that different techniques and methods are implored to analyze a specific social network platform [61-63]. This presents a great challenge hence the need to avoid fragmentation of the social graph implementation and exploit other open cross-platform interactions that will benefit social network graphs for future social media networking. [64]. Other interesting areas of research in social networks include social network modelling, analysis, and mining. The analysis is used "to determine if virtual social networking is tightly bounded diversified or constricted, to find its density and clustering, and to study how the behavior of network members is affected by their positions and connections" [65].

\subsection{Biological Networks}

Complex networks provide a framework for analyzing and investigating biological networks. The structures include regulatory networks, protein-protein interaction, genetic interactions, and metabolic networks. Biological networks represent "a simplified model that describes the interrelationships between a set of functional entities such as genes, proteins, or metabolites" [66]. The studies conducted in [67-69] show a common property such as scale-free degree distribution, small average shortest path length between two nodes, modular structure, and local clustering properties. The authors empirically evaluated the average clustering coefficient of 43 different organisms which shows a highly natural potential modularity and also an independent size of clustering coefficient in metabolic networks. Finally, in determining the ability of metabolic networks to thrive in a dynamically changing environment, there is the need to understand the relationship between the topologies (scale-free, small-world, and hierarchical modularity) and robustness; these features enhance the robustness of metabolism [70-73]. Another biological network that has been studied over the past year is the protein-protein interaction that allows proteins to be represented as a node and the physical interactions as links [74-79]. All these independent works undertaken by various researches significantly contributed to the body of knowledge on a biological network. Other biological networks include food webs [80,81], and neural networks. Based on these reviews conducted, it can be concluded that biological networks share common properties. Although these networks share common properties, Moreno et al. [82] identified a common trend in all the previous articles presented which involves analyzing the structures of all the networks without investigating the dynamics of these systems. They, therefore, analyzed these systems using the various literature on network analysis with nonlinear dynamics models. The authors made an important 
recommendation by asking researchers to provide a common platform that can integrate the various subsystems of biological networks to arrive at more realistic experimental models.

\section{LITERATURE SURVEY}

An extensive literature review has been conducted on complex networks in various databases with high impact factors. A search in databases like IEE, ACM Digital, Springer, Elsevier, Nature and several others show various techniques and application of complex network in the real world, social networks, information network, and technological networks and biological networks [6]. Ellwardt et al. [83] investigated whether complex personal networks have any relationship with higher cognitive functioning in old age. Stronger engagement of older adults in social activities and greater embeddedness in networks is often argued to buffer cognitive decline and lower risks of dementia. It is believed that when older people interact with other people the brain becomes trained thereby enhancing the functioning of their cognitive. Their findings show that older adults reporting greater numbers of relationship types in their network were characterized by higher cognitive capacities both in global and in specific terms than their counterparts reporting fewer types. Importantly, a reduction in network complexity was related to a decline in cognitive functioning. These findings were neither explained by the size of the personal network nor by the presence of specific relationship types in the network, which supports the notion that complexity may carry additional benefits based on the mechanism of activated brain reserves. Crainic and Teoder Gabriel [84] also applied the concept in a transportation system; a service network design in freight transportation in which the presented a joint state of the art review of service network design (frequency and dynamic) with modeling efforts and mathematical programming developments for network design to assist tactical planning of operations. Li. et al. [85] analyses complex networks based on the computation of the degree distribution average path length and clustering coefficient complex characteristics of a public transportation system of Shanghai and the robustness of the network on error. Their findings show that the degree distribution of the network follows a power law distribution and the public transportation network of the city is a network with short average path length and high clustering coefficient as well as the network displaying a very high degree of robustness against error but shows low robustness against attack. Albert [86] study complex networks in relation to WWW, their findings show that the web forms a small world network as the average diameters of the web is made up of only ninety (19) links, in effect any two randomly chosen documents on the web are on average ninety clicks away from each. Fernanda Strozzi and Claudia Colicchia [87] measured the robustness, vulnerability, or resilience in supply chain, they used Google Scholar as the citation network analyzer. The citation network used by the group is a direct network, if an article A cites articles B, there is exist an arrow from B to A represents a flow of knowledge from B to A. The cluster analysis was used for the citation network and Fruchterman- Reingold algorithm was employed as an energy-based method to represent the network and also to identify the clusters after results are obtained compared using the random walk algorithm in [88]. Different types of structures were observed in analyzing the topology of the citation network after using about 345 nodes in their experiment. This includes the star clusters, connection nodes, clusters representing research groups such as the virtual center of super networks, clusters with more centers, and chains. Ana Babus [89] analyzed the financial network, stock exchange-correlation network i.e. stock ownership network, banks credit card network that allows banks to exchange money daily to meet the liquidity demands of customers as well as meeting the international monetary policies. The study was to ascertain whether banks form networks that are resilient to the propagation of small distinct stocks; the results affirm that banks respond to risk by forming links. Costa et al [90] Investigated the concept in relation to epidemiology and anatomy, i.e. the spreading of infections caused by virus or bacteria and the bone structure in mammal respectively The result shows that the channel branching density resembles a power-law implies the existence of distribution hubs, the conditional node degree density indicates a clear tendency of connection between nodes with degrees and the application of the hierarchical clustering coefficient allows identification of typical scales of channel redistribution. A similar application of this concept is evident in the work of [91] where they identified the weakness and ubiquities in complex network, however, emphasized that a concept is an important tool for the creation of hub, robustness, and modularity. A recent work in [92], applied the concept in the brain analysis. Their findings show a better understanding of the correlation between network structure and the process taking place in these networks in particular and synchronization process as well as providing scenarios of how complex networks might respond to different types of damage. Furthermore, complex networks can be applied in epidemiology and anatomy, i.e. the spreading of infections caused by a virus or bacteria and the bone structure in mammal respectively [90, 93]. The result shows that the channel branching density resembles a power-law implies the existence of distribution hubs, the conditional node degree density indicates a clear tendency of connection between nodes with degrees and the application of the hierarchical clustering coefficient allows identification of typical scales of channel redistribution. In another study, Sun et al. [94] on spreading targets in complex networks aims to investigate the spreading ability of nodes towards localized targets in complex networks. The local path algorithm was employed for the problem; the findings of the experiment indicate that the technique proposed outperforms the traditional method especially when the target nodes are relatively few and strongly localized. Finally, complex network can be applied in a semantic network. Semantic network usually encodes the relationship between subjects of words of a language. Semantic network can also be obtained from word association experiments when someone sequentially and freely provides words that are semantically related. A practical application is demonstrated in [95]. This study clearly indicates the semantic networks frequently exhibit small-world and scaler free network model properties with a small world showing high clustering coefficient and low average shortest path while the scalefree model shows a power-law degree distribution.

There is a need for thorough testing before the live execution of a complex Network application technique. A number of novel techniques for evaluating the performance of complex networks have been proposed by various researchers and scholars. $[1,7,8,9,10]$. These techniques can be divided into two main components; modeling and measuring [10-13]. Modelling has two approaches namely analytical and simulation [2, 3, 4, 5, 6]. Whereas Analytical model is a set of equations that describe a computer network efficiency, Simulation is the emulation of the operation of an 
actual live system or process [14]. Classical approaches to network performance evaluation in particular have typically depended on efforts to describe the dynamics of network components over a discrete space-state. After the advent of complex networks, the use of discrete-event simulation packages as an assistance to complex network modeling and performance evaluation is still on the rise $[15,16]$. Even though, Simulators provide a realistic Network representation it depends on how comprehensive they are. The more comprehensive the findings will be the more precise. The accuracy is compensated for with the effort of execution and the complexity of the algorithm. This paper presents a list of some popular Network Simulators available to researchers by reviewing the available discrete event simulation tools, emphasizing the features of the simulators and proposing optimizations for improved performance evaluation outcomes.

\section{PERFORMANCE EVALUATION OF COMPLEX NETWORKS}

There is a need for thorough testing before the live execution of a complex Network application technique. A number of novel techniques for evaluating the performance of complex networks have been proposed by various researchers and scholars. [96, 97, 98, 99,100]. These techniques can be divided into two main components; modeling and measuring [105- 108]. Modelling has two approaches namely analytical and simulation [97, 99,101]. Whereas Analytical model is a set of equations that describe a computer network efficiency, Simulation is the emulation of the operation of an actual live system or process [109]. Classical approaches to network performance evaluation in particular have typically depended on efforts to describe the dynamics of network components over a discrete spacestate. After the advent of complex networks, the use of discrete-event simulation packages as assistance to complex network modeling and performance evaluation is still on the rise $[110,111]$. Even though, Simulators provide a realistic Network representation it depends on how comprehensive they are. The more comprehensive the findings will be the more precise. The accuracy is compensated for with the effort of execution and the complexity of the algorithm. This paper presents a list of some popular Network Simulators available to researchers by reviewing the available discrete event simulation tools, emphasizing the features of the simulators and proposing optimizations for improved performance evaluation outcomes.

\subsection{NS2 (Network Simulator version 2)}

NS2 is the most frequently used kind of object-oriented network simulator which is a discrete event simulator capable of simulating all types of distinct IP networks, creating some network protocols, such as TCP and UDP [112]. It was mainly intended to simulate routing algorithms, multicast, and TCP / IP protocols for the network study community. Hence, its source code is accessible for inspection and modification by any user. The NS-2 simulations are developed using the programming languages $\mathrm{C} / \mathrm{C}++$ and Otcl. It promotes traffic allocation modelling; however, a number of simulation process nodes may hold back the process. Hence, NS2 may not be appropriate for evaluating complex systems. Even though the recent release is more appropriate for sensor networks, as it contains energy modeling [113]. The main drawback of NS-2 is that the tool called Nam provides inferior graphical output.

\subsection{OMNET++ (Objective Modular Network Test bed in $\mathrm{C}++$ )}

OMNET ++ is another a widely used discrete event simulator [114 graphical interfaces. Its other features include Statistical graphic display, each module can be observed in a distinct window, and four distinct speeds of performance. Specifically, it has a very strong graphical interface. It has a lengthy simulation and large memory utilization, consequently, OMNET++ works slowly. Another disadvantage compared to other simulators is the absence of protocols and energy modeling in its library.]. Message animation is one of the primary characteristics of its

\subsection{OPNET (Optimized Network Engineering Tool)}

OPNET is a commercial, discrete event network simulator. It is commonly used for the performance modeling and assessment of local and wide area networks. It includes tools for analyzing and interpreting output information [116]. An extensive model library, modular model creation, strong modeling details and a customizable display of simulation outcomes are be the key strengths of OPNET. Apart from it being costly, the categorization of its variables is not very clear.

\subsection{NetSim}

NetSim is an event simulation network tool used in the testing and study of network laboratories. [117]. It offers metrics of network performance at different abstraction levels such as network, sub-network, node, and a comprehensive packet trace. It has a built-in analytical structure that compares efficiency with graphical alternatives in intra and inter- protocol. Also, its protocol libraries for user enhancement are accessible as open C code. One important limitation of NetSim is that it is a single process discrete simulator of events. Thus, a single event queue is used for the simulation that includes one entry for each station on the network at any specified moment.

\section{Recommendations for Discrete Event Simulation}

Generally, simulating discrete occurrences is often timeconsuming due to the increasing complexity of telecommunications networks and the need for a big amount of observations to produce precise outcomes. Therefore, running different parallel replications (MRIP) on a number of pcs concurrently to reduce the simulation time length is recommended [115]. This paper recommends that Complex Network researchers should create optimized models that are valid and reliable. There is enough literature to suggest that using analytical models helps to address scalability problems such as enabling "what if" analysis to be performed rapidly when assessing system efficiency. Since the source code is available the NAM tool in NS2 can be improved by integrating with a modern and advanced graphical tool. To assist decrease the number of simulations and evaluate the simulation outcomes, statistical methods should be used. The final outcome of a simulation research must also be regarded in context as modeling only produces approximate responses. The estimated features are provided by the random number generators used to drive most models. Statistics should be used as an output interpretation instrument.

\section{CONCLUSION}

The work reviews various network models, theoretical tools, and applications in social networks, biological networks, 
transportation, etc. Complex network is a powerful computational intelligence that has become an essential component in the field of neurobiology, genomics, ecology, business, etc. This review paves way for more research into the dynamics of complex networks based on small-world, scale-free, random network growth rules, and the various optimization principles. The paper also presented and made recommendations to optimize the performance evaluation of complex network models using discrete-event simulators.

\section{REFERENCES}

[1] X. F. Wang and G. Chen, "Complex networks: smallworld, scale-free and beyond," IEEE Circuits and systems magazine, vol. 3, pp. 6-20, 2003

[2] D. B. West, Introduction to graph theory vol. 2: Prentice hall Upper Saddle River, 2001.

[3] Euler, "Historical Notes," Substance P. Raven Press, New York, NY, pp. 1-3, 1977

[4] B. Mohar and C. Thomassen, Graphs on surfaces vol. 2: JSTOR, 2001.

[5] S. Boccaletti, V. Latora, Y. Moreno, M. Chavez, and D.-U. Hwang, "Complex networks: Structure and dynamics," Physics Reports, vol. 424, pp. 175-308, 2006.

[6] M. E. Newman, "The structure and function of complex networks," SIAM review, vol. 45, pp. 167-256, 2003.

[7] G. Chen, X. Wang, and X. Li, Introduction to complex networks: models, structures, and dynamics: Higher Education Press, 2012

[8] D. J. Watts and S. H. Strogatz, "Collective dynamics of 'small-world'networks," Nature, vol. 393, pp. 440-442, 1998.

[9] H. Wang and Y. Guo, "Consensus on scale-free network," in American Control Conference, 2008, 2008, pp. 748-752.

[10] A.-L. Barabási and R. Albert, "Emergence of scaling in random networks," Science, vol. 286, pp. 509-512, 1999.

[11] S. N. Dorogovtsev and J. F. Mendes, "Evolution of networks," Advances in Physics, vol. 51, pp. 1079$1187,2002$.

[12] J. B. Holthoefer, "Semantic networks and cognitive dynamics," Universitat Rovira i Virgili, 2011.

[13] X. F. Liu and C. K. Tse, "A General Framework for Complex Network Applications," arXiv preprint arXiv:1507.05687, 2015.

[14] J. P. Bagrow, Analysis, and applications of complex networks, 2008.

[15] L. Lü and T. Zhou, "Link prediction in complex networks: A survey," Physica A: Statistical Mechanics and its Applications, vol. 390, pp. 1150-1170, 2011.

[16] R. Pastor-Satorras and A. Vespignani, Evolution and structure of the Internet: A statistical physics approach: Cambridge University Press, 2007.

[17] S. Milgram, "The small world problem," Psychology Today, vol. 2, pp. 60-67, 1967.
[18] C. Korte and S. Milgram, "Acquaintance networks between racial groups: Application of the small world method," Journal of personality and social psychology, vol. 15, p. 101, 1970.

[19] K. Klemm and V. M. Eguiluz, "Growing scale-free networks with small-world behavior," Physical Review E, vol. 65, p. 057102, 2002.

[20] S. Wasserman and K. Faust, Social network analysis: Methods and applications vol. 8: Cambridge University press, 1994

[21] S. Achard and E. Bullmore, "Efficiency and cost of economical brain functional networks," PLoS Comput Biol, vol. 3, p. e17, 2007. [22] Q. K. Telesford, K. E. Joyce, S. Hayasaka, J. H. Burdette, and P.

[22] J. Laurienti, "The ubiquity of small-world networks," Brain connectivity, vol. 1, pp. 367-375, 2011.

[23] V. Latora and M. Marchiori, "Efficient behavior of small-world networks," Physical review letters, vol. 87 p. 198701, 2001.

[24] A.-L. Barabási, "Scale-free networks: a decade and beyond," science, vol. 325, pp. 412-413, 2009.

[25] S. Tao, S. Wang, Y. Jiang, and L. Zhu, "Self-similarity Research on Scale-free Networks," in Networking, Sensing and Control, 2008. ICNSC 2008. IEEE International Conference on, 2008, pp. 429-432.

[26] L. Zhang, M. Small, and K. Judd, "Exactly scale-free scale-free networks," Physica A: Statistical Mechanics and its Applications, vol. 433, pp. 182-197, 2015.

[27] L. A. Adamic and B. A. Huberman, "Power-law distribution of the world wide web," Science, vol. 287, pp. $2115-2115,2000$.

[28] R. Cohen and S. Havlin, "Scale-free networks are ultrasmall," Physical review letters, vol. 90, p. 058701, 2003.

[29] Erd6s and A. Rényi, "On the evolution of random graphs," Publ. Math. Inst. Hungar. Acad. Sci, vol. 5, pp. 17-61, 1960.

[30] M. Boguná, R. Pastor-Satorras, and A. Vespignani, "Absence of epidemic threshold in scale-free networks with degree correlations," Physical review letters, vol. 90, p. 028701, 2003.

[31] R. Van Der Hofstad, "Random graphs and complex networks," Available on http://www. win. tue. nl/rhofstad/NotesRGCN. pdf, p. 11, 2009.

[32] M. E. Newman, "Random graphs as models of networks," arXiv preprint condmat/0202208, 2002.

[33] E. N. Gilbert, "Random graphs," The Annals of Mathematical Statistics, vol. 30, pp. 1141-1144, 1959.

[34] M. Penrose, Random geometric graphs: Oxford University Press, 2003

[35] B. Bollobás and O. Riordan, "Coupling scale-free and classical random graphs," Internet Mathematics, vol. 1, pp. 215-225, 2004.

[36] P. Erdos and A. Rényi, "On the evolution of random graphs," Bull. Inst. Internet Statist, vol. 38, pp. $343-$ 347, 1961. 
[37] M. E. Newman, S. H. Strogatz, and D. J. Watts, "Random graphs with arbitrary degree distributions and their applications," Physical review E, vol. 64, p. 026118, 2001

[38] J. E. Hopcroft and R. M. Karp, "An $n^{\wedge} 5 / 2$ algorithm for maximum matchings in bipartite graphs," SIAM Journal on computing, vol. 2, pp. 225-231, 1973.

[39] G. Robins, P. Pattison, Y. Kalish, and D. Lusher, "An introduction to exponential random graph $\left(\mathrm{p}^{*}\right)$ models for social networks," Social networks, vol. 29, pp. 173$191,2007$.

[40] T. A. Snijders, "Markov chain Monte Carlo estimation of exponential random graph models," Journal of Social Structure, vol. 3, pp. 1-40, 2002.

[41] D. S. Callaway, M. E. Newman, S. H. Strogatz, and D. J. Watts, "Network robustness and fragility: Percolation on random graphs," Physical review letters, vol. 85, p. 5468,2000 .

[42] L. Cao, "Data science: a comprehensive overview," Submitted to ACM Computing Survey, pp. 1-37, 2016.

[43] J. Ellson, E. Gansner, L. Koutsofios, S. C. North, and G. Woodhull, "Graphviz - open source graph drawing tools," in International Symposium on Graph Drawing, 2001, pp. 483-484.

[44] E. R. Gansner, "Drawing graphs with Graphviz," Technical report, AT\&T Bell Laboratories, Murray, Tech. Rep2009.

[45] P. Shannon, A. Markiel, O. Ozier, N. S. Baliga, J. T. Wang, D. Ramage, et al., "Cytoscape: a software environment for integrated models of biomolecular interaction networks," Genome research, vol. 13, pp. 2498-2504, 2003

[46] M. Bastian, S. Heymann, and M. Jacomy, "Gephi: an open source software for exploring and manipulating networks," ICWSM, vol. 8, pp. 361-362, 2009.

[47] W. Lu, X. Li, and Z. Rong, "Global stabilization of complex networks with digraph topologies via a local pinning algorithm," Automatica, vol. 46, pp. 116-121, 2010.

[48] R. Van Den Brink and R. P. Gilles, "Measuring domination in directed networks," Social Networks, vol. 22, pp. 141-157, 2000.

[49] G. Yan, T. Zhou, B. Hu, Z.-Q. Fu, and B.-H. Wang, "Efficient routing on complex networks," Physical Review E, vol. 73, p. 046108, 2006.

[50] R. Olfati-Saber and R. M. Murray, "Consensus problems in networks of agents with switching topology and time-delays," IEEE Transactions on automatic control, vol. 49, pp. 1520-1533, 2004.

[51] R. Guimera, M. Sales-Pardo, and L. A. N. Amaral, "Modularity from fluctuations in random graphs and complex networks," Physical Review E, vol. 70, p. 025101, 2004

[52] L. d. F. Costa, O. N. Oliveira Jr, G. Travieso, F. A Rodrigues, P.R. Villas Boas, L. Antiqueira, et al., "Analyzing and modeling real- world phenomena with complex networks: a survey of applications," Advances in Physics, vol. 60, pp. 329-412, 2011.
[53] C. Li, H. Wang, W. De Haan, C. Stam, and P. Van Mieghem, "The correlation of metrics in complex networks with applications in functional brain networks," Journal of Statistical Mechanics: Theory and Experiment, vol. 2011, p. P11018, 2011.

[54] J. Brody, "Rebels, Deviants, and Individualists... Reactions to Psychology: An Evolutionary Ap-proach. By Steven Gaulin \& Donald McBurney, 2001, Upper Saddle River, NJ: Prentice Hall, 13 chapters, 402 pages."

[55] F. Liljeros, C. R. Edling, L. A. N. Amaral, H. E. Stanley, and Y. Åberg, "The web of human sexual contacts," Nature, vol. 411, pp. 907-908, 2001.

[56] X. Guardiola, R. Guimera, A. Arenas, A. Diaz-Guilera, D. Streib, and L. Amaral, "Macro-and micro-structure of trust networks," arXiv preprint cond-mat/0206240, 2002.

[57] M. Boguñá, R. Pastor-Satorras, A. Díaz-Guilera, and A. Arenas, "Models of social networks based on social distance attachment," Physical review E, vol. 70, p. 056122, 2004

[58] P. Zakharov, "Diffusion approach for community discovering within the complex networks: LiveJournal study," Physica A: Statistical Mechanics and its Applications, vol. 378, pp. 550-560, 2007.

[59] P. M. Gleiser and L. Danon, "Community structure in jazz," Advances in complex systems, vol. 6, pp. 565573,2003

[60] C. Nextmedia, "Social Networks Overview: Current Trends and Research Challenges," European Commission Information Society and Media, 2010.

[61] T. G. Armstrong, V. Ponnekanti, D. Borthakur, and M. Callaghan, "LinkBench: a database benchmark based on the Facebook social graph," in Proceedings of the 2013 ACM SIGMOD International Conference on Management of Data, 2013, pp. 1185- 1196.

[62] Y. Yamaguchi, T. Takahashi, T. Amagasa, and H. Kitagawa, "Turank: Twitter user ranking based on usertweet graph analysis," in International Conference on Web Information Systems Engineering, 2010, pp. 240253.

[63] J. Ugander, B. Karrer, L. Backstrom, and C. Marlow, "The anatomy of the facebook social graph," arXiv preprint arXiv:1111.4503, 2011.

[64] A. Bonato and Y. Tian, "Complex networks and social networks," in Advances in Network Analysis and its Applications, ed: Springer, 2012, pp. 269-286.

[65] A. V. Nefian and M. H. Hayes III, "Hidden Markov models for face recognition," choice, vol. 1, p. 6, 1998.

[66] W. W. Goh, Y. H. Lee, M. Chung, and L. Wong, "How advancement in biological network analysis methods empowers proteomics," Proteomics, vol. 12, pp. 550563,2012

[67] H. Jeong, B. Tombor, R. Albert, Z. N. Oltvai, and A.-L. Barabási, "The large-scale organization of metabolic networks," Nature, vol. 407, pp. 651-654, 2000.

[68] R. Overbeek, N. Larsen, G. D. Pusch, M. D’Souza, E. Selkov Jr, N. Kyrpides, et al., "WIT: integrated system 
for high-throughput genome sequence analysis and metabolic reconstruction," Nucleic acids research, vol. 28, pp. 123-125, 2000.

[69] D. A. Fell and A. Wagner, "The small world of metabolism," Nature biotechnology, vol. 18, pp. 1121$1122,2000$.

[70] J. Zhao, H. Yu, J. Luo, Z. Cao, and Y. Li, "Complex networks theory for analyzing metabolic networks," Chinese Science Bulletin, vol. 51, pp. 1529-1537, 2006.

[71] R. Albert, H. Jeong, and A.-L. Barabási, "Error and attack tolerance of complex networks," nature, vol. 406, pp. 378-382, 2000.

[72] J. Stelling, U. Sauer, Z. Szallasi, F. J. Doyle, and J. Doyle, "Robustness of cellular functions," Cell, vol. 118, pp. 675-685, 2004.

[73] H. Kitano, "Biological robustness," Nature Reviews Genetics, vol. 5, pp. 826-837, 2004

[74] J.-C. Rain, L. Selig, H. De Reuse, V. Battaglia, C. Reverdy, S. Simon, et al., "The protein-protein interaction map of Helicobacter pylori," Nature, vol. 409, pp. 211-215, 2001.

[75] L. Giot, J. S. Bader, C. Brouwer, A. Chaudhuri, B. Kuang, Y. Li, et al., "A protein interaction map of Drosophila melanogaster," science, vol. 302, pp. 1727 1736, 2003

[76] V. Colizza, A. Flammini, A. Maritan, and A. Vespignani, "Characterization and modeling of protein-protein interaction networks," Physica A: Statistical Mechanics and its Applications, vol. 352, pp. $1-27,2005$

[77] A. Wagner and D. A. Fell, "The small world inside large metabolic networks," Proceedings of the Royal Society of London B: Biological Sciences, vol. 268, pp. 1803-1810, 2001

[78] S. Wuchty, "Interaction and domain networks of yeast," Proteomics, vol. 2, pp. 1715-1723, 2002.

[79] J.-F. Rual, K. Venkatesan, T. Hao, T. HirozaneKishikawa, A. Dricot, N. Li, et al., "Towards a proteome-scale map of the human protein-protein interaction network," Nature, vol. 437, pp. 1173-1178, 2005.

[80] J. Cohen, F. Briand, and C. Newman, Community food webs: data and theory vol. 20: Springer Science \& Business Media, 2012.

[81] J. M. Montoya and R. V. Solé, "Small world patterns in food webs," Journal of theoretical biology, vol. 214, pp. 405-412, 2002.

[82] Y. Moreno, L. M. Floría, and J. Gómez-Gardeñes, "Current trends in the modeling of biological networks," in From Physics to Biology: the interface between experiment and cumputation(AIP Conference Proceedings), 2006, pp. 150-161.

[83] L. Ellwardt, T. G. Van Tilburg, and M. J. Aartsen, "The mix matters: Complex personal networks relate to higher cognitive functioning in old age," Social Science \& Medicine, vol. 125, pp. 107-115, 2015.
[84] T. G. Crainic, "Service network design in freight transportation," European Journal of Operational Research, vol. 122, pp. 272-288, 2000.

[85] Y. LI, W. ZHOU, and S.-j. GUO, "An Analysis of Complexity of Public Transportation Network in Shanghai [J]," Systems Engineering, vol. 1, p. 006, 2007.

[86] R. Albert, H. Jeong, and A.-L. Barabási, "Internet: Diameter of the world-wide web," Nature, vol. 401, pp 130-131, 1999.

[87] F. Strozzi and C. Colicchia, "Literature review on complex network methods applied to measure robustness in supply chain design," Liuc Papers, 2012.

[88] P. Pons and M. Latapy, "Computing communities in large networks using random walks," in International Symposium on Computer and Information Sciences, 2005, pp. 284-293.

[89] A. Babus, "The formation of financial networks," The RAND Journal of Economics, vol. 47, pp. 239-272, 2016.

[90] L. d. F. Costa, F. A. Rodrigues, G. Travieso, and P. R. Villas Boas, "Characterization of complex networks: A survey of measurements," Advances in physics, vol. 56, pp. 167-242, 2007.

[91] G. Lima-Mendez and J. van Helden, "The powerful law of the power law and other myths in network biology," Molecular BioSystems, vol. 5, pp. 1482-1493, 2009.

[92] J. C. Reijneveld, S. C. Ponten, H. W. Berendse, and C. J. Stam, "The application of graph theoretical analysis to complex networks in the brain," Clinical Neurophysiology, vol. 118, pp. 2317-2331, 2007.

[93] L. da Fontoura Costa, M. P. Viana, and M. E. Beletti, "Complex channel networks of bone structure," Applied physics letters, vol. 88, p. 033903, 2006.

[94] Y. Sun, L. Ma, A. Zeng, and W.-X. Wang, "Spreading to localized targets in complex networks," Scientific Reports, vol. 6, p. 38865, 2016.

[95] M. Steyvers and J. B. Tenenbaum, "The Large- scale structure of semantic networks: Statistical analyses and a model of semantic growth," Cognitive science, vol. 29, pp. 41-78, 2005.

[96] Anastasi, Giuseppe, Alessio Falchi, Andrea Passarella, Marco Conti, and Enrico Gregori. "Performance measurements of motes sensor networks." In Proceedings of the 7th ACM international symposium on Modeling, analysis and simulation of wireless and mobile systems, pp. 174-181. ACM, 2004.

[97] C. Intanagonwiwat, R. Govindan, D. Estrin, "Directed diffusion: a scalable and robust communication paradigm for sensor networks", Proceedings of the International Conference on Mobile Computing and Networking (Mobicom 2000), August 6 - 11, 2000, Boston (MA)

[98] Y. Sankarasubramaniam, O. Akan, I. Akyildiz, "ESRT: Event-to- Sink Reliable Transport in Wireless Sensor Networks" Proc. of the ACM MobiHoc Conference, Annapolis, Maryland, June 2003. 
[99] C. Wan, A. Campbell, L. Krishnamurthy, "PSFQ: A Reliable Transport Protocol for Wireless Sensor Networks," First Workshop on Sensor Networks and Applications (WSNA), September 2002, Atlanta, GA.

[100] C. Wan, S. B. Eisenman, A.T. Campbell, "CODA: Congestion Detection and Avoidance in Sensor Networks," ACM SenSys, 2003.

[101] A. Woo, D. Culler, "A transmission control scheme for media access in sensor networks", Proceedings of the International conference on Mobile Computing and Networking (Mobicom 2001), Rome (Italy) [18] D. S. Bernstein, Z. Feng, B. N. Levine, and S. Zilberstein. Adaptive Peer Selection. In Proc. of Second International Workshop on Peer-to-Peer Systems, Berkeley, CA, USA, 2003. Springer, LNCS 2735

[102] M. Ripeanu, I. Foster, and A. Iamnitchi. Mapping the Gnutella Network: Properties of large-scale peer-topeer systems and implications for system design. IEEE Internet Computing, 6(1):50- 57, jan-feb 2002.

[103] S. Saroiu, P. K. Gummadi, and S. D. Gribble. A Mesaurement Study of Peer-to-Peer File Sharing System. In Proceedings of Multimedia Computing and Networking (MMCN) 2002, Jan 2002.

[104] D. Ganesan, B. Krishnamachari, A. Woo, D. Culler, D. Estrin, and S. Wicker. Complex Behavior at Scale: An Experimantal Study of LowPower Wireless Sensor Networks. Technical Report CSD-TR 02-0013, UCLA.

[105] S. Saroiu, P. K. Gummadi, and S. D. Gribble. A Mesaurement Study of Peer-to-Peer File Sharing System. In Proceedings Of Multimedia Computing and Networking (MMCN) 2002, Jan 2002

[106] M. Ripeanu, I. Foster, and A. Iamnitchi. Mapping the Gnutella Network: Properties of large-scale peer-topeer systems and implications for system design. IEEE Internet Computing, 6(1):50- 57, jan-feb 2002.

[107] D. Stutzbach and R. Rejaie. Characterizing Churn in Peer-to-Peer Networks. Technical report, 2005. Technical Report CIS-TR- 2005-03 University of Oregon
[108] D. Ganesan, B. Krishnamachari, A. Woo, D. Culler, D. Estrin, and S. Wicker. Complex Behavior at Scale: An Experimental Study of LowPower Wireless Sensor Networks. Technical Report CSD-TR 02-0013, UCLA.

[109] Banks J., Carson J., Nelson B., \& Nicol D. (2001). Discrete- Event System Simulation. Prentice Hall. 3 ISBN 0-13-088702-1.

[110] Zhao, Yu, et al. "Fault Modeling and Simulation Based on Cyber Physical System in Complex Distribution Network." 2018 China International Conference on Electricity Distribution (CICED). IEEE, 2018.

[111] Nayyar, Anand, and Valentina Emilia Balas. "Analysis of simulation tools for underwater sensor networks (UWSNs)." International Conference on Innovative Computing and Communications. Springer, Singapore, 2019.

[112] Fall, K. and Varadhan, K. The ns Manual (formerly ns Notes and Documentation). The VINT project, 47.2015. Available at: https://www.isi.edu/nsnam/ns/doc/ns_doc.pdf [Last Access: May 8, 2019]

[113] Korkalainen, M., Sallinen, M., Kärkkäinen, N. and, Tukeva, P., "Survey of wireless sensor networks simulation tools for demanding applications". In proc. of the Fifth International Conference on Networking and Services (ICNS'09). April 20-25, 2009. Valencia, Spain (pp. 102-106). DOI: 10.1109/ICNS.2009.75

[114] Omnet++ Network simulator. Available at: https://www.omnetpp.org/ [Last Access: May 8, 2019]

[115] E. Mota, A. Wolisz, and K. Pawlikowski, "A perspective of batching methods in a simulation environment of multiple replications in parallel " presented at the 32nd Conference on Simulation Orlando, Florida 2000, pp. 761-766.

[116] The NetSim, Available at http://en.wikipedia.org/wiki/NetSim. [Last Access: May 8, 2019] 